\title{
MULTI-BATCH SLIP STACKING IN THE MAIN INJECTOR AT FERMILAB
}

\author{
K. Seiya, T. Berenc, B. Chase, J. Dey, P. Joireman, I. Kourbanis, J. Reid \\ FNAL, Batavia, IL 60510, U.S.A.
}

\begin{abstract}
The Main Injector (MI) at Fermilab is planning to use multi-batch slip stacking scheme in order to increase the proton intensity at the NuMI target by about a factor of 1.5.[1] [2] By using multi-batch slip stacking, a total of 11 Booster batches are merged into 6, 5 double ones and one single. We have successfully demonstrated the multibatch slip stacking in MI and accelerated a record intensity of $4.6 \mathrm{E} 13$ particle per cycle to $120 \mathrm{GeV}$. The technical issues and beam loss mechanisms for multibatch slip stacking scheme are discussed.
\end{abstract}

\section{INTRODUCTION}

The slip stacking scheme has been used in MI for antiproton production since December 2004. Two batches from the Booster with the intensity of 4.5E12 ppp each are combined into one batch in the MI with the slip stacking scheme. By this, the intensity for antiproton production is increased to $8.5 \mathrm{E} 12 \mathrm{ppp}$.

The MI also accelerates proton beam to the NuMI beam line for the MINOS neutrino experiment. In the "mixed mode" cycle, the first two Booster batches are merged with the slip stacking. Five additional Booster batches and the one merged batch are accelerated together. One doubled batch goes to anti proton production and 5 single ones go to the NuMI target. In the "NuMI only" cycle, all 7 batches, one doubled and 5 single batches were accelerated and sent to the NuMI.

For the Proton Plan the beam intensity to the NuMI will be increased by a factor of 1.5 using multi-batch slip stacking. A total of 11 batches will be injected from Booster and slip stacked. The intensity of injection batch will be $4.3 \mathrm{E} 12 \mathrm{ppp}$ and the total intensity at extraction is expected to be 4.5E13 ppp of which 3.7E13 ppp will be sent to the NuMI and 8.0E12 ppp to the anti proton production in the mixed mode cycle, assuming $5 \%$ beam loss.

\section{MULTI BATCH SLIP STACKING}

Two rf systems with different frequencies are used for the 11-batch slip stacking. Five batches are injected on the central frequency of the first rf system, captured with $100 \mathrm{kV}$ rf voltage and decelerated. The second $\mathrm{rf}$ has been off during the 5 batch injections. After the frequency of the first system has lowered, the $6^{\text {th }}$ batch is injected on the central frequency of the second rf system and captured with $100 \mathrm{kV}$ rf voltage. Since the $6^{\text {th }}$ injection batch and the other five have different frequencies, they move on different orbits in the ring and slip each other. After the batch train slipped by about one batch length, the 7th batch was injected on the central frequency. Figure 1 shows the slipping frequencies, total rf voltage and beam intensity in the MI. After the $10^{\text {th }}$ batch injection, both frequencies are moved up to around the central frequency to wait for the higher and lower energy batches lined up longitudinally, then bunches are captured with $1 \mathrm{MV}$ rf voltage with one central frequency.

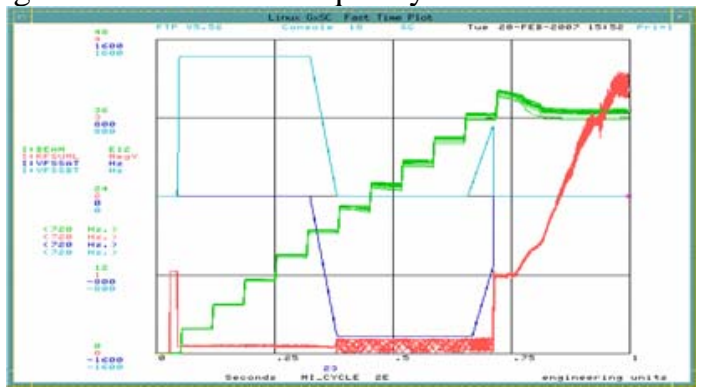

Figure 1: The frequency functions for the first (blue) and the second (cyan) rf, the total rf voltage (red), and the beam intensity (green) in the MI.

\section{STATUS}

We have been doing beam studies for the 11-batch slip stacking since 2005 and have verified that the scheme works for both the mixed mode and NuMI only cycles. Figure 2 and 3 are WCM signals at injection and showing the 11 batch slip stacking process from the $1^{\text {st }}$ batch injection to the time before acceleration on NuMI only and mixed mode cycles. NuMI only beam was sent with an intensity of 4.0E13 ppp and an efficiency of 93\% (Figure 4). The mixed mode cycle beam was sent with intensity of 6.5E12 ppp for antiproton production and 2.2E13 ppp to the NuMI, but there was beam loss at extraction kicker for the antiproton production. The MI also accelerated the record intensity of 4.6E13 ppp to 120 $\mathrm{GeV}$ to the beam dump. We are working on beam loss issues to increase the efficiency to greater than $95 \%$ and to localize energy and location of the beam loss.

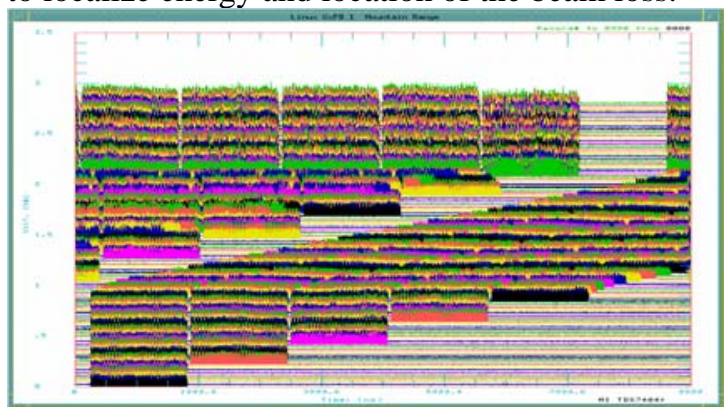

*Operated by Fermilab Research Alliance, LLC under Contract No. DE-AC02-07CH11359 with the United States Department of Energy. "kiyomi@fnal.gov 
Figure 2: Mountain range plot showing 11 batch slip stacking process in the Numi only cycle. Horizontal scale is $10 \mu \mathrm{sec}$ and revolution is $\sim 11 \mu \mathrm{sec}$.

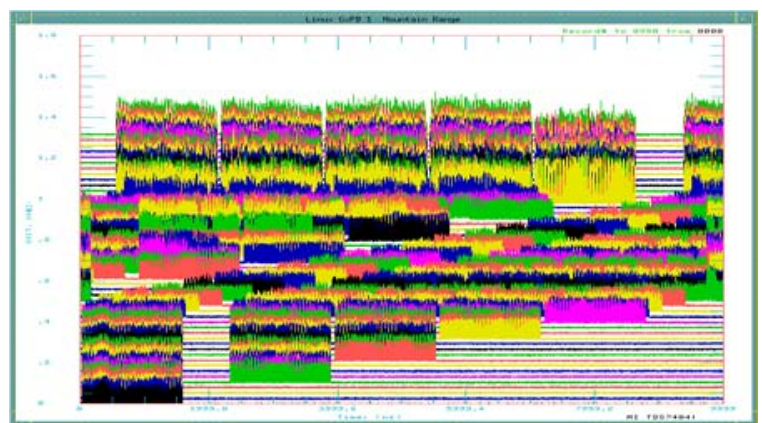

Figure 3: Mountain range plot showing 11 batch slip stacking process in the mixed mode cycles.

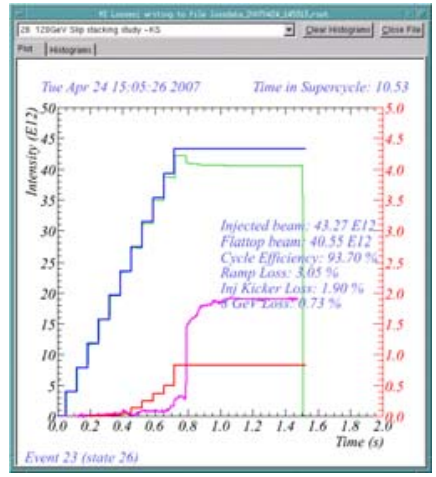

Figure 4: The intensity from the Booster (blue) and the total intensity in the MI (green) for a NuMI only cycle.

\section{CYCLE EFFICIENCY AND BEAM LOSS}

There were four types of beam losses, injection kicker loss, ramp loss, extraction kicker loss and lifetime loss, during the cycle. In the following sections we are going to explain the mechanism of beam loss with measurements and simulation results.

\section{Injection kicker loss}

Figure 4 shows the beam intensity from the Booster and the total intensity in the MI. The intensity from the Booster was measured with a DC current transformer (DCCT) located on the beam transport line between the Booster and the MI, while the total MI intensity was measured with a DCCT in the MI. The MI total intensity after injection was lower than the intensity from the Booster and there was beam loss at the injection kicker location. This showed that the injection kicker voltage kicked out particles circulating in the MI.

Longitudinal simulations were carried out to understand the process of the beam loss located on the injection kicker gap. Two different frequencies were applied to one bunch which was filling rf bucket of $100 \mathrm{kV}$, but still all particles were in the bucket. One higher frequency by + $1425 \mathrm{~Hz}$ and a central frequency rf were applied to the bunch. Figure 5 (left) shows particles in the phase space after $67 \mathrm{msec}$. Particles around the bucket separatrix were pushed to the left hand side below transition energy. Lower frequency was applied in Figure 5 (right) and particles were pushed to the right hand side. The batches from the $6^{\text {th }}$ to $10^{\text {th }}$ were injected on the central frequency while the first 5 batches were slipping longitudinally with lower frequency so that particles outside the rf bucket were kicked out by the injection kicker voltage. More detailed simulation for 11 batch injections has been done and results were shown in Figure 6 injection by injection. The six pictures show the phase space just before the next batch injection. There were particles on the gap where the next batch was going to be injected in.
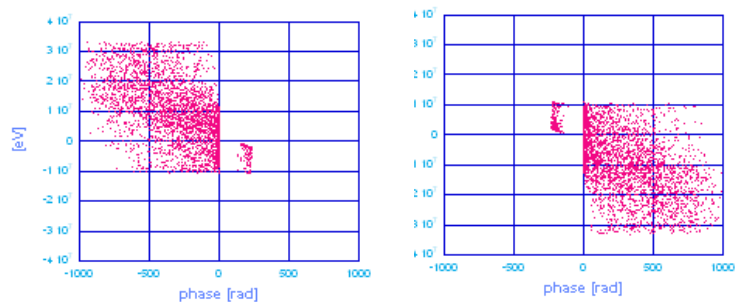

Figure 5: Simulation results with one bunch. Horizontal scale is \pm 160 buckets and vertical scale is $+/-40 \mathrm{MeV}$.

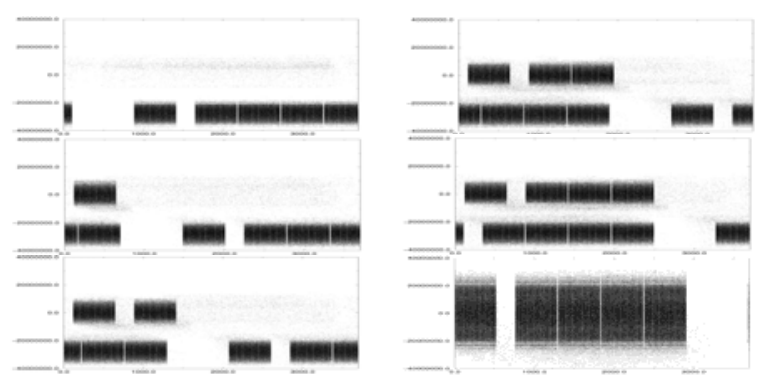

Figure 6: Simulation for 11 batch injections showing the phase space just before the next batch injection for the 5th to the 10 th batches. Vertical scale is $+/-40 \mathrm{MeV}$.

\section{Ramp loss}

There is beam loss at $0.8 \mathrm{sec}$ in Figure 4. It happens at the energy of $9 \mathrm{GeV}$ after the beginning of acceleration. The 11 batches which have gone through slip stacking were accelerated to $9.5 \mathrm{GeV}$ in the longitudinal simulation. Figures 7 shows longitudinal phase spaces before acceleration and at the energy of $9.5 \mathrm{GeV}$. The MI momentum aperture was measured at $8.9 \mathrm{GeV}$ and it was $\pm 0.1 \%$, so that the particles with lower energy will hit the aperture and cause beam loss at $9 \mathrm{GeV}$.

The kicker loss and ramp loss were measured with three different intensities and compared with simulation results. Longitudinal phase space distribution was measured at injection with phase space Tomography which used wall current monitor signals. This is shown in Figure 8. The projections to phase and energy were fit to Gaussian functions to estimate the bunch length and energy spread for the simulation. The intensity was 1.77E12, 2.65E12 and 3.44E12 ppp from Booster. Both bunch length and energy spread was large with high intensity. The 
simulation results were shown in Figure 9 and injection kicker loss on the measurement agrees with simulation but the measured ramp loss is $~ 2$ times larger than the simulation. This is probably because of transverse effects which were not taken into account in the simulation. Both losses were larger when the emittance was large. Smaller beam size from the Booster is essential for high efficiency.

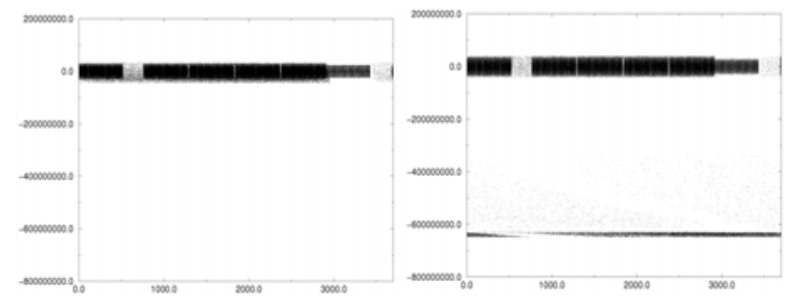

Figure 7: Longitudinal phase spaces before acceleration and at the energy of $9.5 \mathrm{GeV}$ from the simulation.

Vertical scale is from $-800 \mathrm{MeV}$ to $200 \mathrm{MeV}$

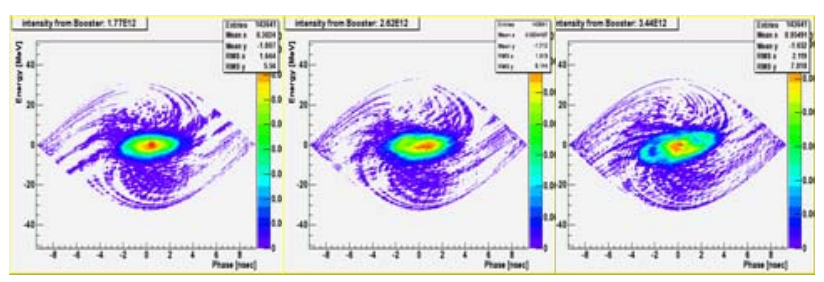

Figure 8: Longitudinal phase space distribution from phase Tomography measurements. Vertical scale was +/$40 \mathrm{MeV}$ and Horizontal scale was +/- 9 nsec.

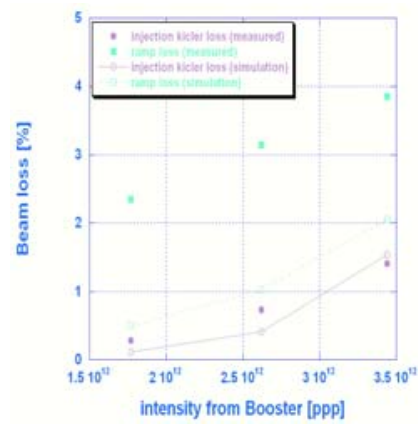

Figure 9: Measurements and Simulation results for injection kicker and ramp losses with different intensities.

\section{Extraction kicker loss}

After beam was accelerated in the simulation there were particles in the gap between the batch to antiproton production and the one to the NuMI beam line. The batch to the antiproton production would be extracted earlier than the batches to NuMI target so that the particles in the gap were kicked by the extraction kicker voltage. WCM signals were measured at extraction in the beam studies and there were bunches in the gap. Figure 10 shows the mountain range plot with WCM signals. The left and right edge of antiproton production batch was zoomed in vertically in the lower figures. A beam loss tripped radiation monitor on the transport line to the antiproton ring during machine studies. On the current mixed mode operation, the MI bunch by bunch damper was used to "anti-damp" the beam in the kicker gap after recapture at $8 \mathrm{GeV}$. We plan to reconfigure the MI damper to work during 11 batch slip stacking. [3]

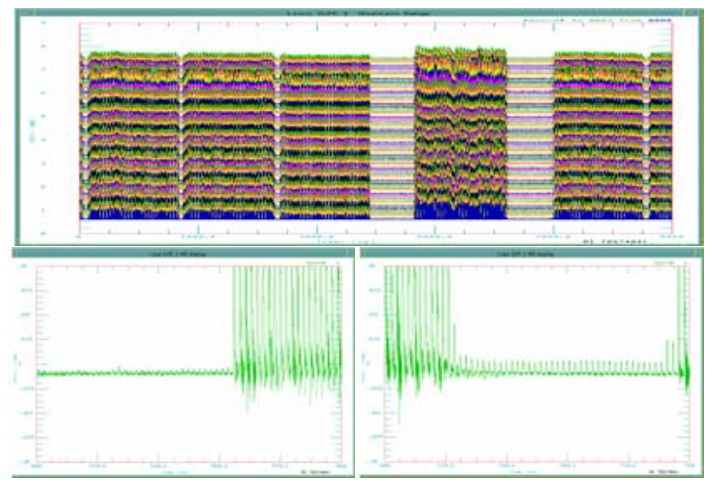

Figure 10: (Upper) Mountain range plot at extraction. (Lower) The left and right edge of batch to antiproton.

\section{Lifetime loss}

There is a small lifetime loss during the 11 batch injection. This is because of the large chromaticity value used to stabilize the beam. We plan to reduce the chromaticity after MI damper operational during slip stacking.

\section{SUMMARY}

We plan to increase the intensity to NuMI by a factor of 1.5 with 11-batch slip stacking. We have been doing beam studies for this scheme and have already verified that it worked for both the mixed mode and NuMI-only cycle. The beam was sent to NuMI target with the intensity of $4.2 \mathrm{E} 13$ and the efficiency of $93 \%$. The MI also accelerated the record intensity of $4.6 \mathrm{E} 13$ to 120 $\mathrm{GeV}$.

There were four types of beam losses, injection kicker loss, ramp loss, extraction kicker loss, and lifetime loss, during the cycle. Each loss was studied with measurements and simulation. The longitudinal beam emittance from the Booster is essential to the injection kicker loss and ramp loss. They were larger with the high intensity. The extraction kicker loss can be cured with the MI damper with anti damping beam at $8 \mathrm{GeV}$. Lifetime loss should be improved with lower chromaticity.

\section{REFERENCES}

[1] I. Kourbanis, "Present and future high energy accelerator for Neutrino experiment”, HB2006, These proceedings.

[2] K. Seiya, et al, "Progress in Slip stacking and barrier bucket”, 2006 ICFA Workshop, Japan, June 2006.

[3] P. Adamson, et al, "Operational performance of a bunch by bunch digital damper in the Fermilab Main Injector”, 2005 PAC, Knoxville, May 2005. 
